\title{
Advanced Characterization of Silica-Encapsulated Aluminum Pigments
}

\author{
Draga Dragnea ${ }^{1,2}$, Dragos Gudovan², Elena Zaharia², Ion Dragnea1', Costin Sorin Bildea2* \\ 1 AlbaAluminiu LTD., Str. Gării 6, 516100 Zlatna, Alba, Romania \\ 2 Department of Chemical and Biochemical Engineering, Faculty of Applied Chemistry and Materials Science, University \\ Politehnica of Bucharest, Str. Gh. Polizu 1-7, 011061 Bucharest, Romania \\ * Corresponding author, e-mail: s_bildea@upb.ro
}

Received: 03 June 2019, Accepted: 04 October 2019, Published online: 28 November 2019

\begin{abstract}
For environmental reasons, the paints industry shifts from solvent-borne towards water-borne formulations. This change is challenging the business of aluminum pigments, as the hydrogen released by the reaction of aluminum with water degrades the optical properties, besides being a safety concern. In this work, industrial-grade aluminum pigments are encapsulated, by a well-known method, in a silica matrix by sol-gel process using isopropanol - a more suitable solvent for the industry. The effectiveness of the encapsulation process is proven by advanced physical methods (Scanning Electron Microscopy, Energy Dispersive X-Ray Analysis, Selected Area Electron Diffraction, Fourier Transformed InfraRed Spectroscopy, Thermo-Gravimetric Analysis) and by industry-relevant tests (stability in water, hiding power, flop and granulometry). Moreover, advanced surface-applied physical methods (High Resolution Transmission Electron Microscopy combined with Selected Area Electron Diffraction and Scanning Transmission Electron Microscopy, and FT-IR microscopy) clearly show the homogeneity of the resulting pigments, a quality which is highly desirable for practical applications. The results demonstrate that stability comparable to that of pigments passivized by chromium-based inhibitors is easily achieved, for a variety of operating conditions. However, accomplishing a homogeneous silica layer of the right thickness is the determining factor for good optical properties.
\end{abstract}

Keywords

water-borne coatings, Aluminum Pigments, sol-gel process, physical characterization, stability

\section{Introduction}

Aluminum Pigments (AP) are used in solvent-borne metallic paints due to their optical properties [1], with wide applications in automotive and motorcycle industry, general industrial coatings, printing inks $[2,3]$, and decorative and plastic industry [4]. Aluminum Pigments are obtained by milling of atomized aluminum powder with mineral spirit and fatty acids [5]. The surface of the Aluminum Pigment particles is covered by a thin layer of oxide that, at $\mathrm{pH}$ neutral conditions, protects the pigment against corrosion. In the context of environmental regulations banning large VOC (Volatile Organic Compounds) contents, the paints and pigments industry is developing water-based products $[6,7]$. However, alkaline conditions affect the oxide layer [8]. As aluminum easily reacts with water, large amounts of hydrogen are released, damaging the optical properties of the pigment and leading to serious safety concerns [1]. The problem can be solved by using chromate-based corrosion inhibitors, but this is not an environmentally-friendly solution due to the use of heavy-metal compounds. A sustainable alternative is encapsulating the aluminum flakes in a protective layer [9]. The encapsulation can be achieved with an inorganic layer (usually $\mathrm{SiO}_{2}$ obtained by sol-gel process). The method of encapsulation into the silica shell have been first patented in 1959 [10] and lately improved as described e.g. in [11-13]. Other alternatives use organic layer or hybrid layer with organic-inorganic materials [1, 14]. The sol-gel process is an economic and easy way to form inorganic nanostructures on the aluminum flake surface [15-17]. Sol-gel films offer hydrophobic properties to the substrate, while being an environmentally-friendly alternative to chromate-based surface treatments [16]. The sol-gel process includes hydrolysis and condensation reactions of different kind of precursors, generating an inorganic network around the aluminum flake $[18,19]$. Although tetraethylorthosilicate (TEOS) 
remains the preferred precursor for industrial-scale applications [15], recent works report the formation of a hybrid layer on the aluminum flake surface achieved by using TEOS and $\gamma$-aminopropyl triethoxysilane [20], TEOS and poly-methyl methacrylate-butyl acrylate-(3-methacryloxypropyl)-trimethoxysilane [9], or TEOS and polymer brushes [6]. The inorganic framework can be chemically bound to the aluminum flake or the silica can form a shell around the flake, without the formation of chemical bounds. It is generally accepted that hydroxy-groups on the aluminum flake surface are the anchoring points for the silica sol-gel film [20, 21]. Thus, anchoring agents as $\mathrm{H}_{2} \mathrm{O}_{2}$ or copper-ammonia complexes can be used to oxidize the aluminum surface and to form hydroxy moieties.

In the present work, Aluminum Pigments suitable for water-borne systems were prepared by the well-known sol-gel method using an industrial-grade Aluminum Pigment as raw material, TEOS as precursor, isopropanol as solvent and ammonia as catalyst. Note that isopropanol is a solvent commonly used in industry, in contrast to ethanol, which is more expensive and, in many countries, is subject of severe regulations and taxation. Moreover, we show that removing the organic phase of the pigment by washing with a solvent (an expensive step from the industry point of view) might be omitted without any loss of product quality. In addition, we present detailed characterization by advanced physical methods (Scanning Electron Microscopy - SEM, Energy Dispersive X-Ray Analysis - EDXA, High Resolution Transmission Electron Microscopy - HR-TEM, Selected Area Electron Diffraction - SAED, Fourier Transformed InfraRed Spectroscopy - FT-IR, Thermos-Gravimetric Analysis TGA). The results demonstrate that stability comparable to that of pigments passivized by chromium-based inhibitors can be quite easily achieved. Although similar good stability was demonstrated in the works cited above, from the results presented in the open literature is not obvious that the optical properties are also preserved. Thus, our contribution demonstrates that accomplishing a homogeneous silica layer of the right thickness is the determining factor for good optical properties.

\section{Materials and methods}

Aluminum Pigments (AP) produced by Alba Aluminiu Ltd. [22], a South-East Europe provider, were used as received. TEOS $98 \%$, ammonia solution $25 \%$, ethylene glycol butyl ether (EGBE) $99 \%$ from Sigma Aldrich Ltd. and isopropanol $99.9 \%$ from Chimreactiv Ltd. were used as received. Distilled water was used in the experiments.
In the encapsulation experiments two types of raw material were used, Aluminum Pigments as received from Alba Aluminiu (referred to as sample MP) and washed Aluminum Pigments (referred to as sample MPS). The washing process consists of mixing MP samples with isopropanol at room temperature for several minutes, followed by decanting and drying at $80{ }^{\circ} \mathrm{C}$.

In general, the encapsulation experiments lead to pigments with good stability. However, they had different optical properties, not all of them fulfilling the industry standards. In the following, the best result of encapsulating MPS and MP will be denoted by P1 and P4, respectively. For illustration purposes, some results will be also shown for the sample P9, with excellent stability but with optical properties unsuitable for practical applications.

\subsection{Encapsulation setup}

The AP and TEOS in isopropanol were added to a three-neck round bottom flask which was connected to a reflux-condenser, fitted with a thermometer and a mechanical stirrer. A thermostatic water bath was used to heat the flask at the required temperature. Few minutes after the temperature reached $50{ }^{\circ} \mathrm{C}$, the ammonia solution was added.

The mixture was stirred for 3 hours and the resulting product was separated by decanting and vacuum dried at $80{ }^{\circ} \mathrm{C}$.

It should be emphasized that, in the experiments described by the open literature, the surface of the industrial grade Aluminum Pigment is modified before the encapsulation is performed. Two different methods are used:

1. the pigments are thoroughly washed with a solvent, such as ethanol (for 12 hours) [2], acetone [6], xylenetoluene-MEK (four wash-dry cycles) [14]; and

2. the pigment is activated with $\mathrm{H}_{2} \mathrm{O}_{2}$ [20] or solution containing copper-ammonia complexes [20, 21].

In this work we show that encapsulated pigments with good optical properties can be obtained by omitting this preliminary step (washing or activation), thus using as raw material the pigment as obtained from the milling step. This point is very important for the industry, as it avoids the costs of recovery, purification and reuse of the washing solvent.

\subsection{Stability test}

In industry, the stability is assessed by measuring the amount of hydrogen released by the Aluminum Pigment in contact with water at $60{ }^{\circ} \mathrm{C}$, for 7 days (gassing method). However, this method is time-consuming and therefore less suitable 
for screening purposes. Thus, we set-up a simple method to quickly determine the stability of the encapsulated Aluminum Pigment: encapsulated AP dispersed in EGBE is transferred to a three-necked vessel filled with water and kept at high temperature (for example, $70-98^{\circ} \mathrm{C}$ ). The flask is fitted with a magnetic stirrer, a thermometer, and a reflux-condenser. A tube filled with water is attached to the flask and connected to a storage tank where the water displaced by hydrogen is moved and can be measured. The Aluminum Pigments which were characterized as "stable" by this quick screening method were also "stable" when analyzed according to the industrial method.

\subsection{Optical properties}

The hiding power is the ability of the system to eliminate the color or the color differences of the substrate. Regarding classical pigments, it is commonly described that organic pigments and black pigments exhibit hiding power due to their absorption properties, while inorganic pigments and white pigments exhibit hiding power due to their light scattering properties. The hiding power of AP is due to their ability to reflect the incident light which confers opacity to the system in which they are embedded. In the hiding-power test, the black surface of the test panel is covered with a thin layer $(0.75 \mu \mathrm{m})$ of a standard lacquer in which the Aluminum Pigment is dispersed, and the measured property is the ability of this layer to cover the darkened surface underneath and to confer opacity.

The flop is one of the important parameters of Aluminum Pigments that affects the gloss [5]. The flop represents the alteration of brightness of a surface as a function of the observation angle. The flop can be determined by observing the test panel on face and oblique angles and measuring the reflected light with a multi-angle spectrophotometer (X-rite). The surface of the test panel is illuminated at $45^{\circ}$ (incident light) and the intensity $L$ of the reflected light is measured at $15^{\circ}, 45^{\circ}$ and $110^{\circ}$. Using these values, the flop is calculated as [5]:

$$
F=2.69 \times\left(L_{15}-L_{110}\right)^{1.11} / L_{45}^{0.86} .
$$

Metallic effect pigments obtained by ball milling do not have a defined particle size. The particle size distribution (which can be approximated as Gaussian) has a direct influence on the optical appearance of the metallic effect pigments. One of the methods for determining the particle size distribution of metallic pigments is by laser granulometry method. The particle size distribution determined by this method is characterized by three values: D10, D50,
D90. D10 measures the fine fraction of the particle size distribution and means that $10 \%$ of all measured particles are smaller than the respective value and $90 \%$ are larger. D50 and D90, similarly defined, characterize the average and the coarser fraction of the particle size distribution. The products with narrow particle size distribution are metallic bright and have a good flop [5].

\subsection{Physical characterization}

SEM and HR-TEM [23] give information regarding the shape and size of the particles, and the homogeneity of the surface. As the electron beam interacts differently with different materials, information about the chemistry underneath the surface layer is also provided. Microstructural SEM images were obtained using a Quanta Inspect F 50 instrument, with field emission and fitted with energy dispersive analysis accessory (EDXA). Transmission electron microscopy images were obtained using an electron microscope Titan Themis 200 (80-200 kV FEG) which performs low-temperature analysis - cryo- in (HR)TEM, STEM, SAED modes and 3D tomography in TEM and STEM modes. A small quantity of the powder was dispersed in ethyl alcohol and left in an ultrasonic bath for 15 minutes. After that, a small quantity of a dilute solution was placed on a $\mathrm{Cu}$ grid coated with a $\mathrm{C}$ thin film with holes and allowed to dry for 30 minutes before electron microscopy by transmission.

FT-IR is sensitive towards the vibrational state of chemical bonds. The occurrence of a new chemical bond is reflected by a new distinct vibrational state in the spectra. When one atom becomes involved in hydrogen, van der Walls or dipole interaction, the wavelength of the signal characterizing the bond changes. FT-IR spectra were recorded using a Thermo Scientific iS 50 spectrometer with built-in diamond crystal Attenuated Total Reflectance (ATR) accessory.

The infrared microscopy (complementary to FT-IR) was performed using a Thermo Nicolet iN10 spectrometer fitted with liquid nitrogen cooled mercury cadmium telluride (MCT) detector.

TGA records the thermal behavior of the sample. The thermal derivatograms were obtained using a Netzsch instrument, model type STA Jupiter 449C, using a heating rate of $10 \mathrm{~K} / \mathrm{min}$ under argon inert atmosphere $(50 \mathrm{~mL} / \mathrm{min})$.

\section{Results and discussion}

\subsection{Stability tests}

Fig. 1 (a) shows the amount of hydrogen released by $1 \mathrm{~g}$ of pigment (raw material, MP) in contact with water, at different temperatures. It can be observed that a large 

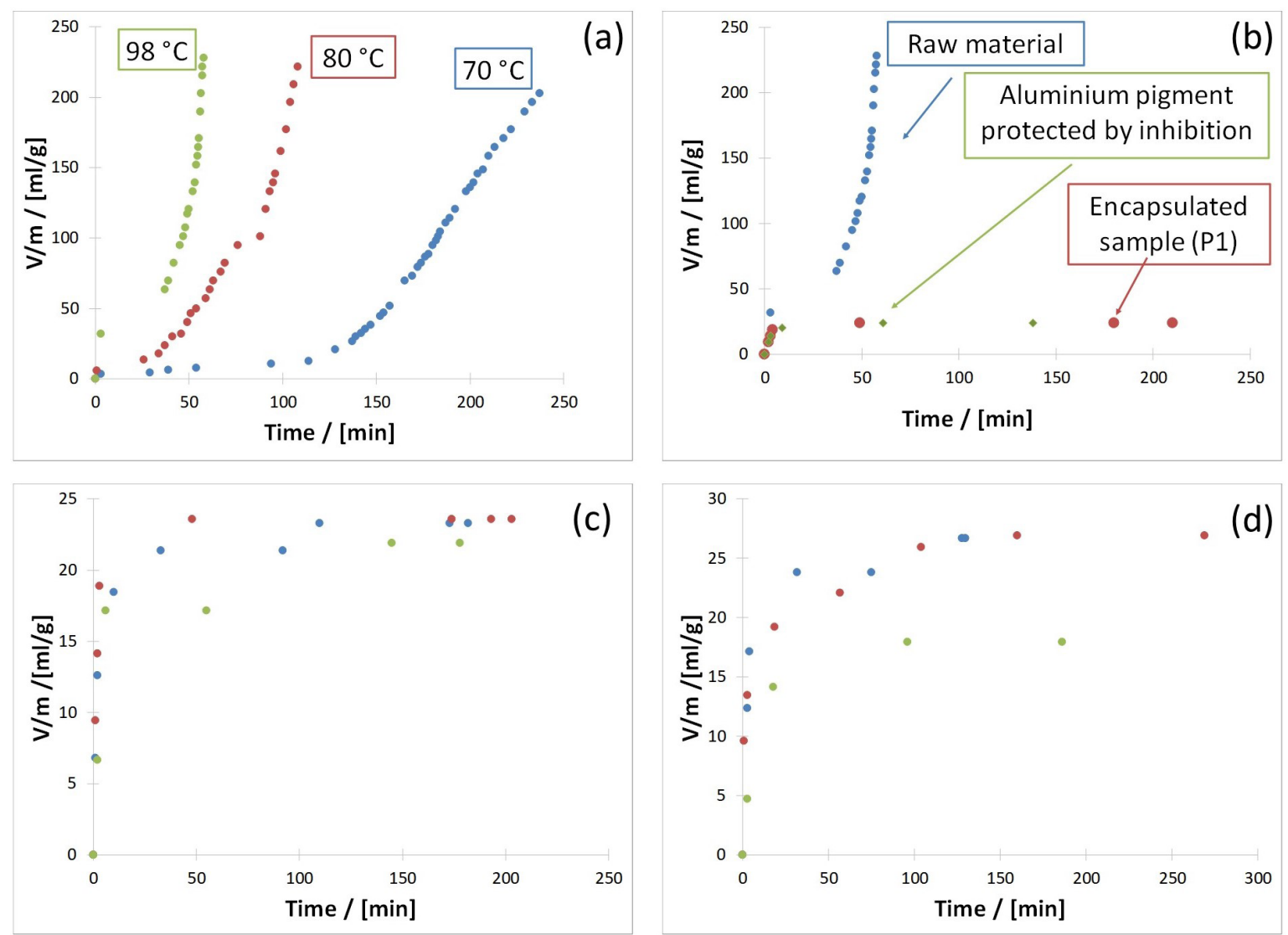

Fig. 1 The stability of raw material at different temperatures (a). The stability of the raw material, and Aluminum Pigment protected by encapsulation and inhibition, at $98^{\circ} \mathrm{C}$ (b). Stability test for P1 (c) and for P4 (d).

quantity of hydrogen is released because the pigment is unstable in water. As expected, the release of hydrogen is faster at higher temperature. At $70{ }^{\circ} \mathrm{C}$ it takes 4 hours to release $200\left[\mathrm{ml} \mathrm{H}_{2}\right] /$ [g AP], while the same amount of hydrogen is released in less than one hour when the test is performed at $98^{\circ} \mathrm{C}$. Fig. 1 (b) presents the stability of one encapsulated sample, which is compared with the raw material and with a commercial pigment protected by inhibition. The encapsulation and chemical inhibition greatly enhanced the stability of the pigment. Fig. 1 (c) and (d) shows results of several stability tests performed on several samples of encapsulated pigments P1 and $\mathrm{P} 4$ obtained in different experiments, proving that the efficient encapsulation is reproducible. The amount of hydrogen evolved during 200 minutes is in the range 15-30 $\left[\mathrm{ml} \mathrm{H}_{2}\right] /$ [g AP]. Comparatively, the result reported in reference [24] is $400\left[\mathrm{ml} \mathrm{H}_{2}\right]$ / [g AP] (stability test performed in $\mathrm{HCl} 1 \mathrm{~mol} / \mathrm{l}$ ), when the encapsulation was performed in propanol. The same reference reports much higher stability (no hydrogen evolved) if the encapsulation is performed in butanol. However, the optical properties of the encapsulated pigments were not investigated.
When the stability was tested in $\mathrm{NaOH} 0.1 \%$ wt. [25], the amount of hydrogen evolved in 400 minutes exceeded $35\left[\mathrm{ml} \mathrm{H}_{2}\right] /$ [g AP]. In this case [25], the pigment was treated with $\mathrm{H}_{2} \mathrm{O}_{2}$ prior to encapsulation. Again, the optical properties of the encapsulated pigment were not investigated.

\subsection{Optical properties}

The hiding power test (Fig. 2) is one of the most important tests conducted in Aluminum Pigments industry [5]. The results of this test show the difference between the
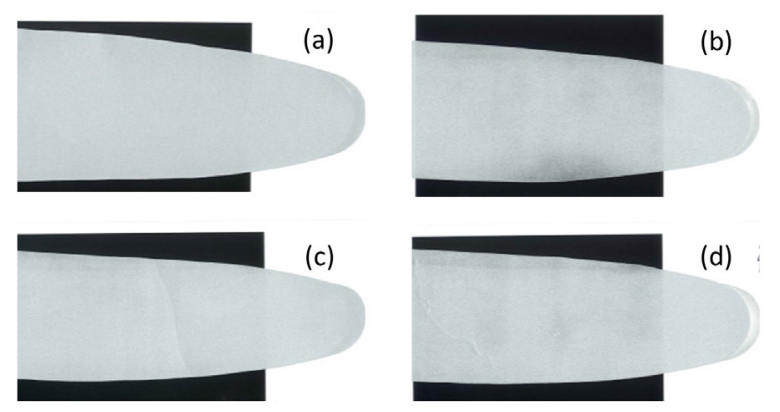

Fig. 2 Hiding power results: raw material MP (a); encapsulated sample P9 lacking hiding power (b); encapsulated samples P1 (c) and P4 (d) with good hiding power. 
capabilities of the raw material and of three encapsulated samples (obtained in encapsulation experiments with different recipes - reaction temperature, amount of TEOS precursor, catalysts) with similar stability but with different hiding power. While the encapsulated pigments denoted by P1 has optical properties like the raw material, a slight degradation is observed in P4. For illustration purposes, the sample P9 which is unsuitable for practical applications, is also shown. Regarding the flop characteristic, it decreases from 17.9 (MP) to 16.9 (P1), 16.2 (P4) and 16.5 (P9). In general, higher flop is better. In our case, the target value is 17.9, the flop of the Aluminum Pigment before encapsulation. For these samples, the $\mathrm{D}_{50}$ characteristic is $29.55,30.50,34.72$ and $33.95 \mu \mathrm{m}$, respectively. The value has increased because of the silica layer deposed on the Aluminum Pigment surface.

\subsection{Physical characterization}

The microstructural aspect of the MPS (raw material, washed with isopropanol) and MP (raw material) samples is shown in scanning electron microscopy images (secondary electron images) in Fig. 3. Note that the images have a resolution up to $400,000 \mathrm{x}$, much higher compared to other micrographs shown in the literature (which have a resolution ranging from $5,000 \times[1,2,26]$ up to $100,000 \times[25]$ ).

The micro-composition analysis by energy dispersive X-ray spectroscopy (EDXA, spectra not shown) revealed that, besides Aluminum, Carbon and Oxygen are present in both samples (MP and MPS), originating from the oily mix used to inhibit the Aluminum Pigment for transportation purposes and the residual left-overs from the washing process.
The microstructural aspect of the P1 and P4 samples is shown in scanning electron microscopy images (secondary electron images) of Fig. 3 ((b), (c) and (e), (f)). The same aspect of the particles can be noticed in both figures. The high-resolution SEM images $(\times 400,000)$ shows that particles measuring between 8 and $15 \mathrm{~nm}$ are present on the surface of the aluminum flakes. Round-shape particles with a tendency to agglomerate in larger aggregates can be observed. Similar results are shown by [1] and [6], although at a much lower resolution.

Agglomerations of particles in flakes and platelets form with nanometric thicknesses can be observed.

The micro-compositional analysis by dispersive X-ray energy spectroscopy (EDXA) highlighted the presence of the main elements $\mathrm{C}, \mathrm{O}, \mathrm{Al}$ and $\mathrm{Si}$ in sample $\mathrm{P} 1$ and $\mathrm{P} 4$. Carbon is still present as an impurity due to the organic oil content and the residual solvent used for washing. The presence of $\mathrm{Si}$ in the sample is due to the inorganic shell formed around the Aluminum Pigment core.

The HR-TEM images in bright field obtained on sample MP shown in Fig. 4 reveal that the sample is composed of very large aluminum sheets consisting of crystalline grains in the range 100-200 $\mathrm{nm}$. The SAED analysis shows crystalline planes with the interplanar distance related to the metallic Al. From the distribution of the elements obtained on the sample MP and shown in Fig. 4, it is observed that the elements identified are $\mathrm{Al}$ and $\mathrm{O}$, also identified with EDXA. Oxygen is found on the surface of the sample, which leads us to say that there is a layer of aluminum oxide of about $10 \mathrm{~nm}$ on the surface of the sample. The sample also has a small amount of organic residue on the surface.
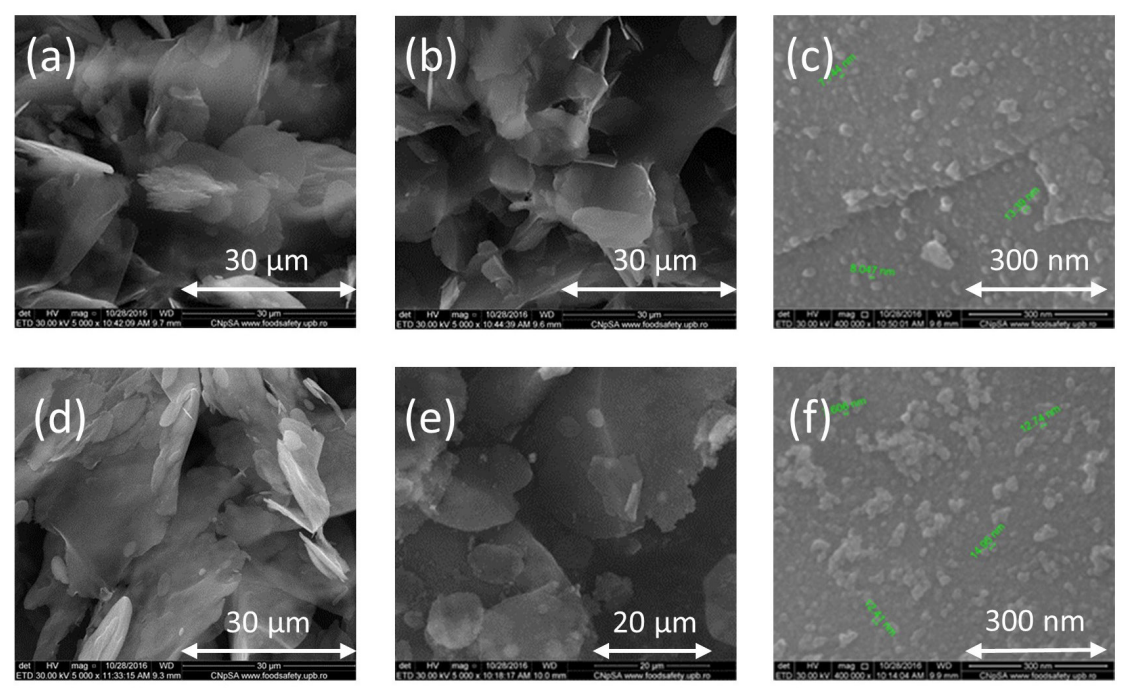

Fig. 3 Microstructural aspect of the MPS sample at $\times 5,000$ (a), P1 ×5,000 (b), P1 ×400,000 (c). Microstructural aspect of the MP sample at $\times 5,000$ (d), $\mathrm{P} 4 \times 5,000$ (e), $\mathrm{P} 4 \times 400,000$ (f) 

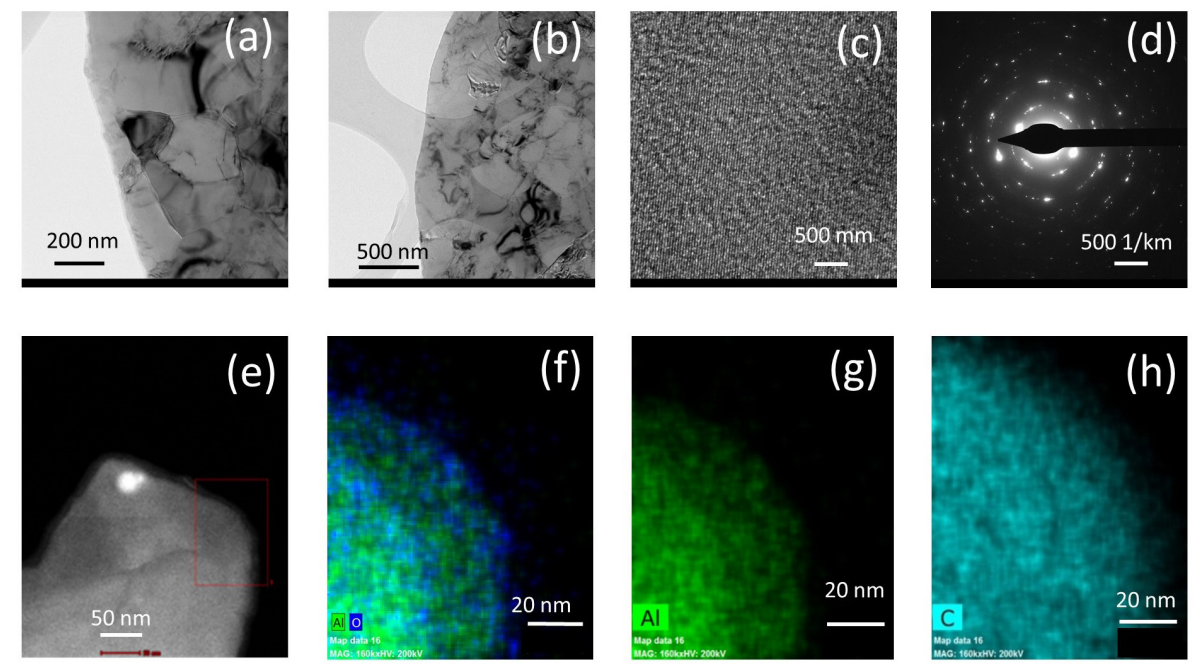

Fig. 4 HR-TEM images in bright field (at different resolutions (a), (b)) and SAED pattern obtained on MP sample ((c), (d)). STEM image detailing where the distribution map was made by elements on sample MP (e) and distributed maps according to elements obtained on sample MP ((f) Al, O; (g) Al; (h) C)

The HR-TEM images in bright field obtained on the MPS sample (Fig. 5) shows that the sample is made up of very large aluminum sheets. SAED analysis shows a high degree of crystallinity, with crystalline planes with the interplanar distance related to the metallic Al.

From the STEM distribution of the elements obtained on the sample MPS (Fig. 5) it is observed that the elements identified are $\mathrm{Al}$ and $\mathrm{O}$. The oxygen is found on the surface of the sample, which leads us to say that on the surface of the sample there is a layer of aluminum oxide of some $\mathrm{nm}$, thinner than in the case of the non-washed AP. The sample also has no noticeable organic residue on the surface.

HR-TEM images in bright field obtained on sample P1 in Fig. 6 (h) shows that the sample is composed of very large aluminum sheets which are coated with a layer of spherical particles and a non-crystalline layer. The layer size is about $10 \mathrm{~nm}$ and the particles have a size of $50-60 \mathrm{~nm}$. The crystalline phase belongs to the aluminum metallic phase and the non-crystalline phase is the silica shell that formed around the metallic core. The shell formed tend to be homogenous in nature and uniformly distributed on the surface of the aluminum particle. In contrast, He et al. [6] report the formation of an uneven layer on silica encapsulated aluminum flakes. From the STEM distribution of the elements obtained on sample P1 and shown in Fig. 6, the identified elements are $\mathrm{Al}, \mathrm{O}$ and $\mathrm{Si}$. Oxygen is found both on the surface of the sample and on the particles identified on the surface of the aluminum. Also, $\mathrm{Si}$ is present at the same locations where $\mathrm{O}$ is present, which leads us to conclude that the layer deposed on aluminum particles is constituted from $\mathrm{SiO}_{2}$.
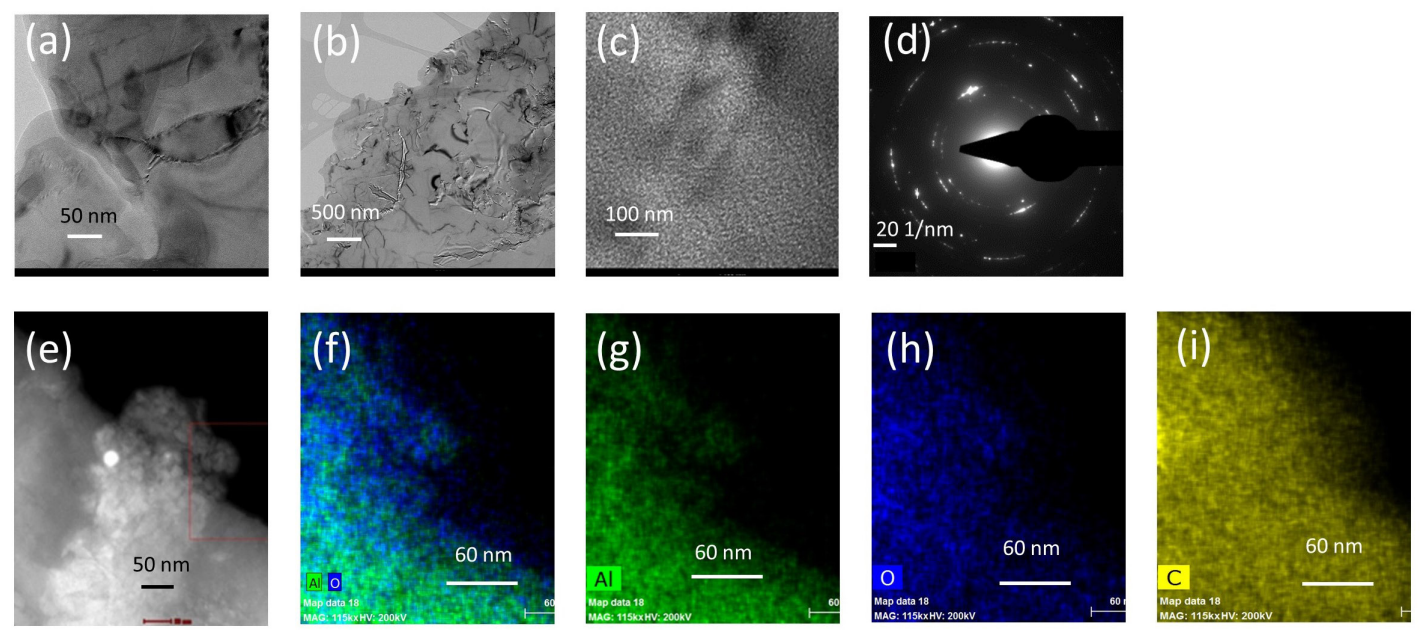

Fig. 5 HR-TEM image in bright field (at different resolutions (a), (b)) and SAED pattern obtained on MPS sample ((c), (d)). STEM image detailing where the distribution map was made by elements on the MPS sample (e) and distributed maps by elements obtained on MPS ((f) Al, O; (g) Al; (h) O; (i) C). 

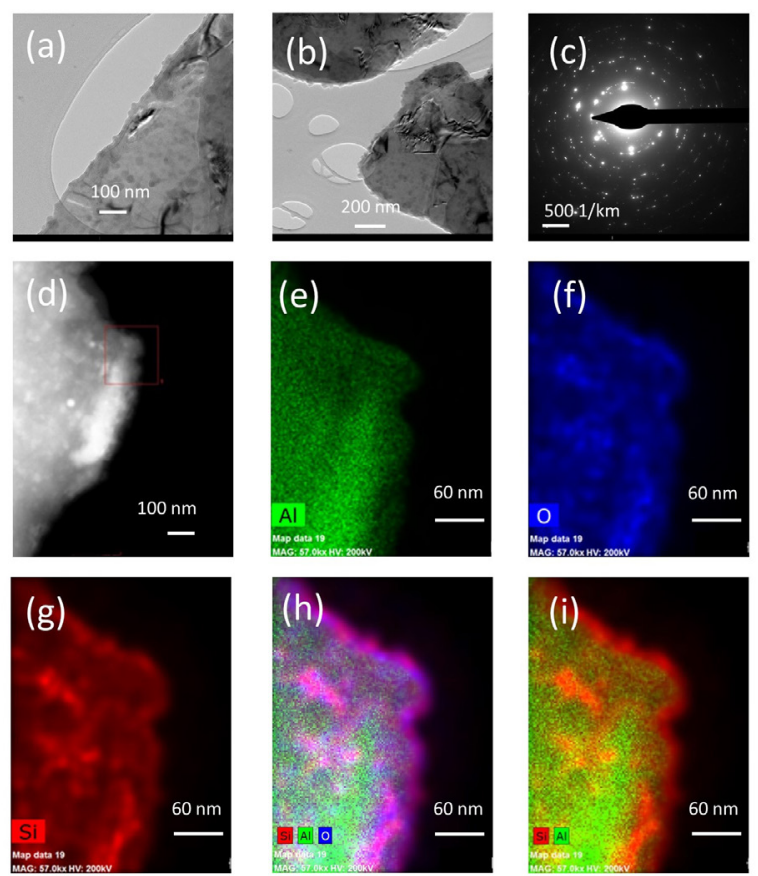

Fig. 6 HR-TEM image in bright field ((a), (b)) and SAED pattern (c) obtained on P1 sample. STEM image detailing where the distribution map was made (d) by elements on the P1 sample and distributed map by elements obtained on P1 ((e) Al; (f) O; (g) Si; (h) Si, Al, O; (i) Si, Al)

HR-TEM images in bright field obtained on sample P4 in Fig. 7 shows that the sample is made of very large aluminum sheets which are coated with a layer of spherical and irregular shaped particles and a non-crystalline layer. The deposed layer is uneven for this sample. The size of the layer is about $20 \mathrm{~nm}$ wide. From the distribution of the elements obtained on sample P4 in Fig. 7 it can be distinguished that the only elements identified are Al, O and Si. Oxygen is found both on the surface of the sample and on the particles identified on the surface of the aluminum. Also, $\mathrm{Si}$ is present at the same locations as $\mathrm{O}$, which leads to the conclusion that the layer deposed on aluminum particles is a $\mathrm{SiO}_{2}$ layer. On this sample, the layer is thinner, and is not uniformly distributed over the large particles of Al.

In the literature, one can also find results of elemental analysis techniques, such as $\operatorname{EDS}[1,2]$ and EDXA [4, 6, 14]. These techniques confirm the existence of silicium on the surface (in addition to $\mathrm{Al}, \mathrm{O}, \mathrm{C}$ ). The images presented in this contribution actually show how these elements are distributed on the surface (this is possible due to the particular mode in which the TEM is operated). Moreover, Zhou et al. [1] estimate the thickness of the coating layer in an indirect manner. In this work, the thickness of the layer can be well seen in Figs. 6 and 7.
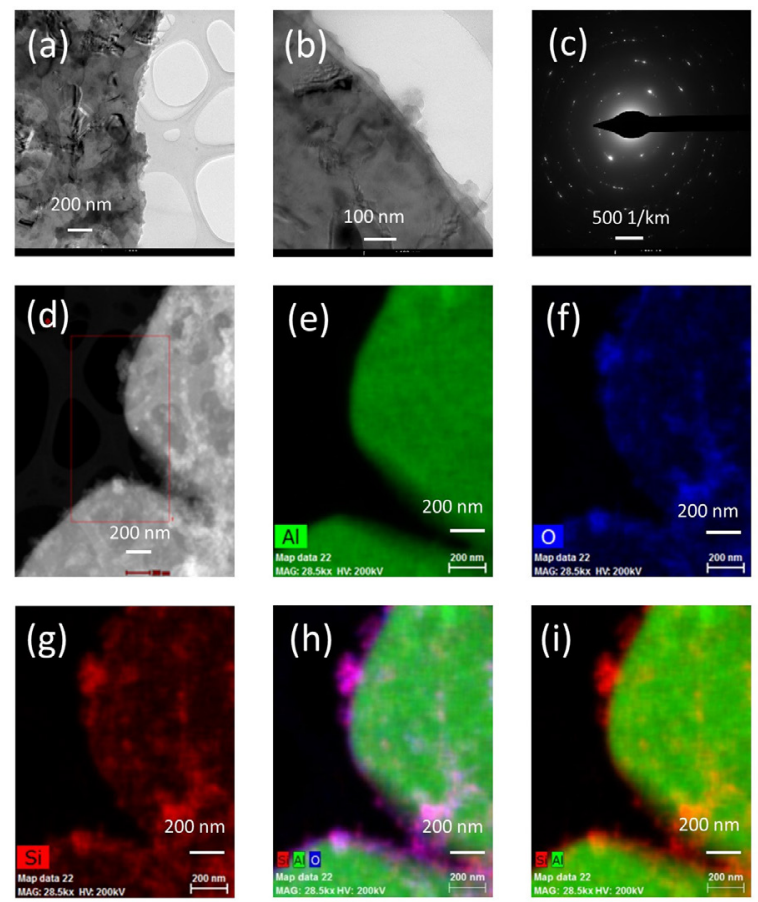

Fig. 7 HR-TEM image in bright field ((a), (b)) and SAED pattern obtained on P4 sample (c). STEM image of the P4 sample, detailing where the distribution map was made (d) and distributed map by elements obtained ((e) Al; (f) O; (g) Si; (h) Si, Al, O; (i) Si, Al)

HR-TEM images in bright field obtained on sample P9 (Fig. 8 (a), (b)) also shows that the sample is made of very large aluminum sheets which are coated with a layer of spherical and irregular shaped particles and a non-crystalline layer and that the deposed layer is uneven. The size of the layer is about $100 \mathrm{~nm}$ wide. From the distribution of the elements obtained on sample P9 in Fig. 8 (d)-(h) it can be distinguished that the only elements identified are $\mathrm{Al}$, $\mathrm{O}$ and $\mathrm{Si}$. Oxygen is found on the surface of the sample. The particles are around $200 \mathrm{~nm}$. Also, Si is present where $\mathrm{O}$ is present, which leads to the conclusion that the layer deposed on aluminum particles is a $\mathrm{SiO}_{2}$ layer.

Recalling the hiding power of the encapsulated samples (P1 - the best; P4 - good; P9 - the worst), we conclude that a $\mathrm{SiO}_{2}$ layer of uniform thickness is essential for achieving good optical properties.

The TGA curves for MP (Fig. 9) show that a sudden, $19 \%$ mass decrease occurs from $50{ }^{\circ} \mathrm{C}$ to $150{ }^{\circ} \mathrm{C}$. This is explained by the presence of oleic acid [9]. On all samples, the mass slightly decreases (by about $1-2 \%$ ) up to $460{ }^{\circ} \mathrm{C}$. From this point to high temperatures $\left(1000^{\circ} \mathrm{C}\right)$, the mass slightly increases (by about $1.5 \%$ for MPS and MP, and $4.5 \%$ for P1 and P4), which can be explained by oxidation reactions. On the unencapsulated Aluminum Pigment 

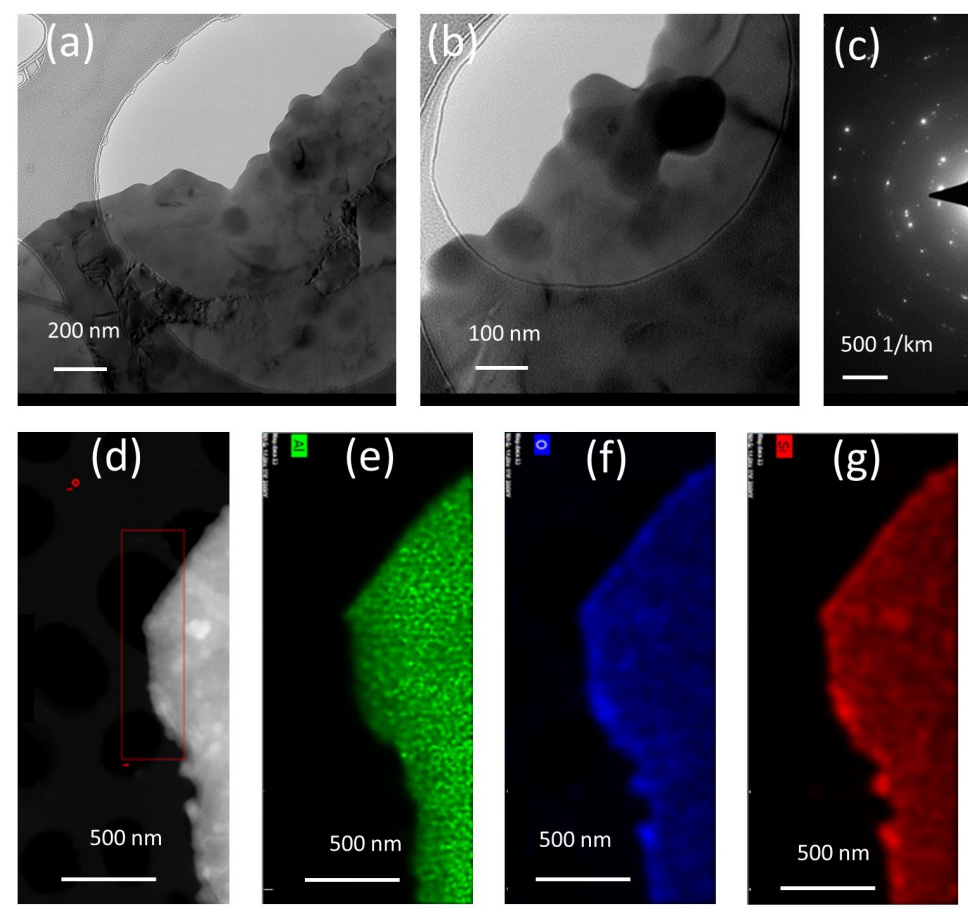

Fig. 8 HR-TEM image in bright field obtained ((a), (b)) and SAED pattern on P9 sample (c). STEM image detailing where the distribution map was

made by elements on the P9 sample (d) and distributed map by elements obtained on P9 ((e) Al; (f) O; (g) Si; (h) Si, Al, O)

(a)

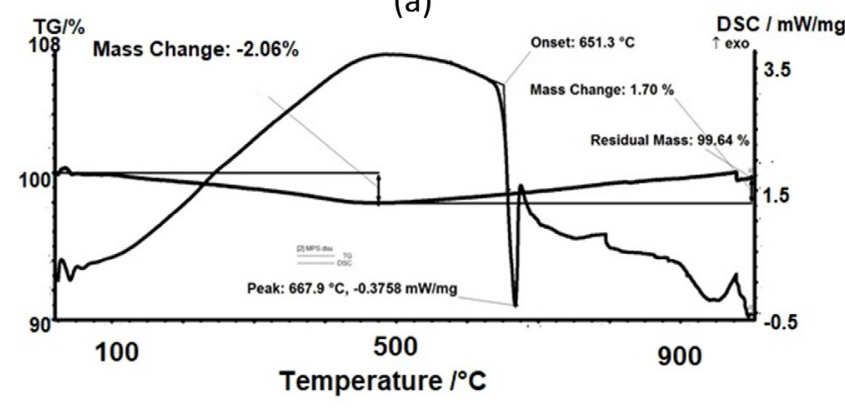

(c)

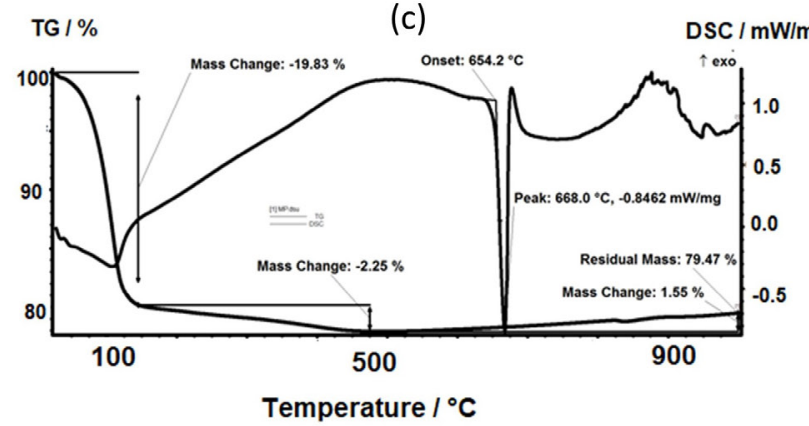

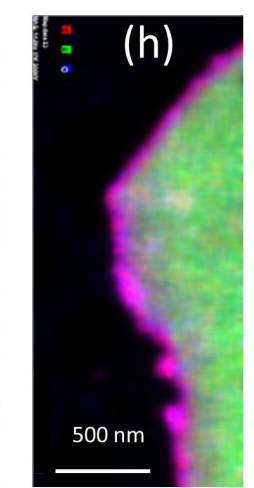

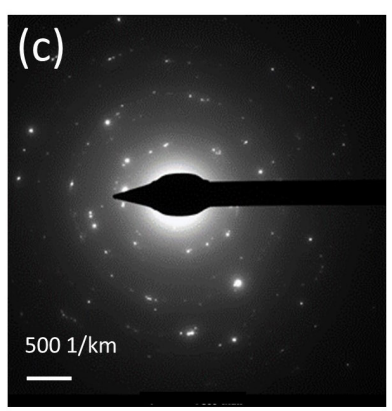

Fig. 9 Comparative TG/DSC curves of raw material and encapsulated pigments. MPS (a) and P1 (b); MP (c) and P4 (d).

samples MP and MPS, DSC shows endothermic peaks with the starting point / peak values of $651^{\circ} \mathrm{C} / 667.9^{\circ} \mathrm{C}$ (MPS), and $654.2^{\circ} \mathrm{C} / 668^{\circ} \mathrm{C}$ (MP), values which are near the melting point of metallic aluminum $\left(660.3{ }^{\circ} \mathrm{C}\right)$. For the encapsulated samples, the endothermic peak is located at significantly lower values $\left(617{ }^{\circ} \mathrm{C} / 639.4{ }^{\circ} \mathrm{C}\right.$ for $\mathrm{P} 1$, and

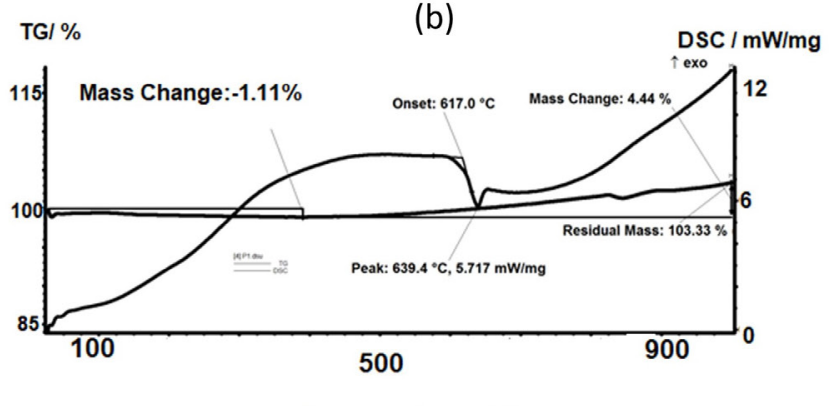

Temperature $/{ }^{\circ} \mathrm{C}$

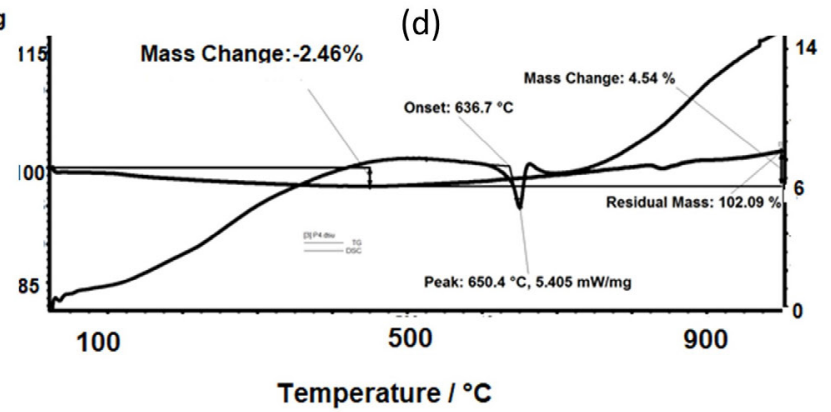

$636.7^{\circ} \mathrm{C} / 650.4^{\circ} \mathrm{C}$ for P4), probably due to formation of a silica - aluminum eutectic mixture.

The FT-IR spectra (not shown) reveal the differences between the encapsulated samples and the raw materials. Besides the Si-OH peak $\left(950 \mathrm{~cm}^{-1}\right)$ [15], the encapsulated samples, $\mathrm{P} 1$ and $\mathrm{P} 4$, exhibit the peaks 
characteristic for Si-O-Si at $1144 \mathrm{~cm}^{-1}$ [27] and for $\mathrm{Si}-\mathrm{CH}_{2}$ at $1239 \mathrm{~cm}^{-1}$ [9] confirming the formation of the silica shell. The IR microscopy is in direct agreement with both FT-IR and STEM results, showing an increased signal on the samples containing a slightly thicker layer of silica. The IR micrographs (Fig. 10, red corresponds to more intense peak) show that the silica peaks are more homogenously distributed across the P1 sample, but the silica layer is rather thin. In the case of the P4 sample, the silica layer is thicker, but the distribution is less homogenous compared to the P1 sample. Again, this agrees with the slightly better optical properties of the sample P1.

\section{Conclusions and perspectives}

The protective technique using a silica shell is a wellknown technique, economical and environmentally responsible, which yields the same results (stability and optical properties) as the commercially available mixtures of Aluminum Pigment and chemical passivizing agents.

Using advanced characterization techniques, our study contributes to a better understanding of the structure of the encapsulated AP.

STEM, infrared microscopy and TG/DSC curves prove the formation of an amorphous silica shell around the aluminum flake. The optical properties of the final pigment are in direct correlation with the homogeneity of the shell. A thicker shell reduces covering properties (hiding power) and gives an improper aspect to the area covered with pigment.

For the Aluminum Pigment tested in this work, the washing of the organic content used during manufacturing of the Aluminum Pigment by milling improves only slightly the optical properties. Thus, a washing step might be avoided in the industrialization of the technique, the raw materials being directly introduced "as is" to the reaction environment. Moreover, the encapsulation process can be carried on in isopropanol, which is a more industry-acceptable solvent compared to ethanol (the usual polar solvent for laboratory experiments). However, as different manufacturers use different stabilizers in the milling process and during storage and transportation, these conclusions should be tested for pigments from other sources.

\section{Acknowledgement}

The financial support of the project "Innovative processes for encapsulation of aluminum pigments in polymer matrices", PN-II-PT-PCCA-2013-4-2069, contract 233/2014 is gratefully acknowledged. (d)

(d)

(d)

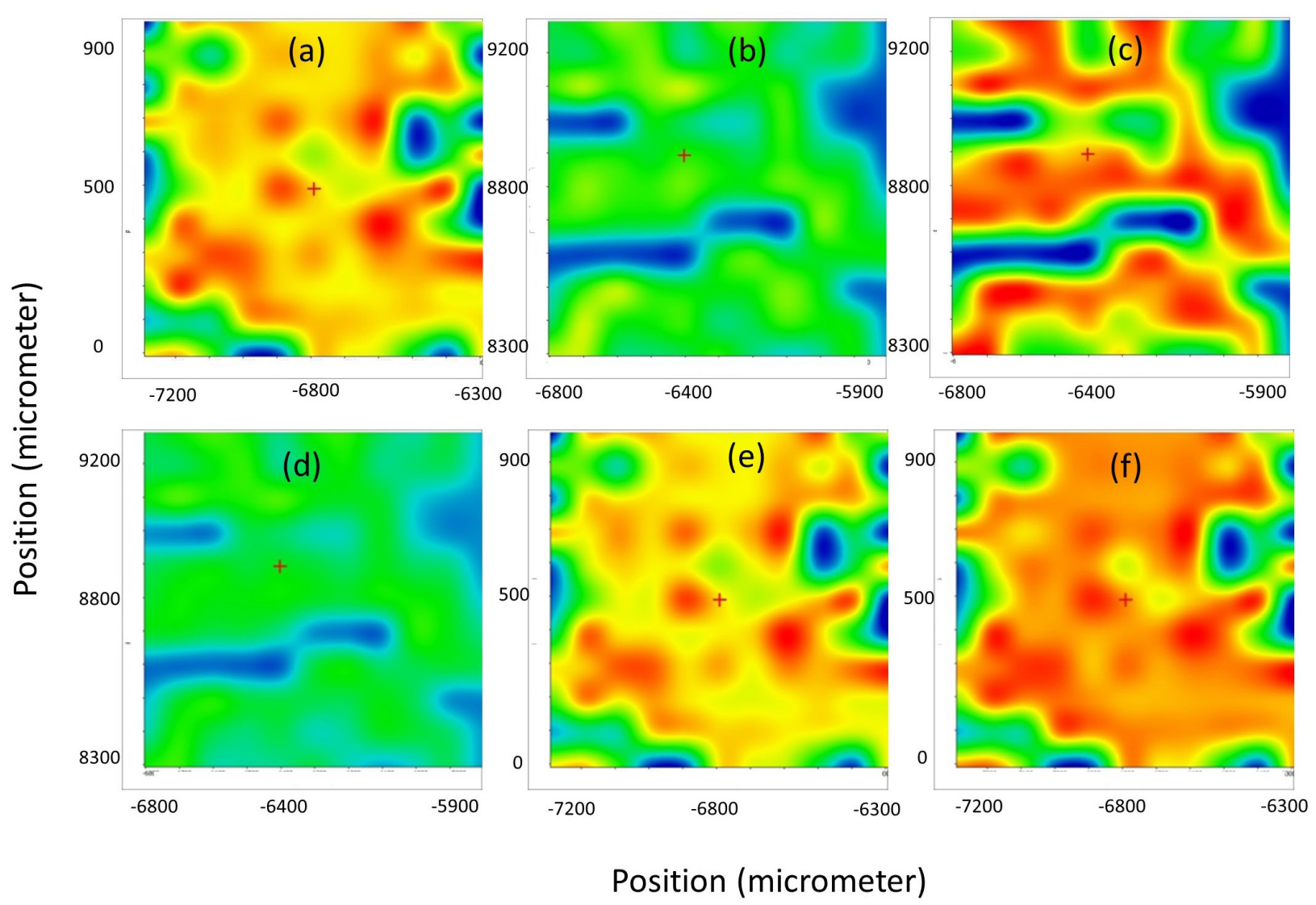

Fig. 10 Infrared microscopy for sample P1 ((a)-(c)) and P4 ((d)-(f)), at $1065 \mathrm{~cm}^{-1}$ ((a), (d)), $1144 \mathrm{~cm}^{-1}$ ((b), (e)), $1239 \mathrm{~cm}^{-1}$ ((c), (f)) 


\section{References}

[1] Zhou, L., Huang, S. L., Kong, J. R., Zhou, T., Zuo, Y. J. "Characterization of flaky aluminium pigments multi-coated by $\mathrm{TiO}_{2}$ and $\mathrm{SiO}_{2}$ ", Powder Technology, 237, pp. 514-519, 2013. https://doi.org/10.1016/j.powtec.2012.12.035

[2] Zhang, Y., Ye, H., Liu, H., Han, K. "Preparation and characterization of aluminium pigments coated with silica for corrosion protection", Corrosion Science, 53(5), pp. 1694-1699, 2011. https://doi.org/10.1016/j.corsci.2011.01.027

[3] Abdulwahab, M., Fayomi, O. S. I., Awe, F. E. "Anti-Corrosion and Passivation Potential of AA6063-type Al-Mg-Si Alloy with Avogadro Natural Oil in $\mathrm{HCl}$ solution", Journal of Advanced Electrochemistry, 2(2), pp. 75-77, 2016. [online] Available at: http://jacsdirectory.com/journal-of-advanced-electrochemistry/ admin/issues/20160427150555_2-2-02\%20JAEC16017\%20published.pdf [Accessed: 02 June 2019]

[4] Amirshaqaqi, N., Salami-Kalajahi, M., Mahdavian, M. "Investigation of corrosion behavior of aluminium flakes coated by polyeric nanolayer: Effect of polymer type", Corrosion Science, 87, pp. 392-396, 2014.

https://doi.org/10.1016/j.corsci.2014.06.045

[5] Wissling, P. "Metallic Effect Pigments: Fundamentals and Applications", Vincentz Network, Hannover, Germany, 2006.

[6] He, Y., Li, H., Ou, L., Ding, F., Zhan, Z., Zhong, Y. "Preparation and characterisation of water-based aluminium pigments with modified $\mathrm{SiO}_{2}$ and polymer brushes", Corrosion Science, 111, pp. 802-810, 2016 https://doi.org/10.1016/j.corsci.2016.06.014

[7] Joubert, M., Save, M., Mornet, S., Lavaud, F., Pellerin, V., Morvan, F., Tranchant, J. F., Duguet, E., Billon, L. "Surface patterning of micro-sized aluminum flakes by seeded dispersion polymerization: Towards waterborne colored pigments by gold nanoparticles adsorption", Polymer, 55(3), pp. 762-771, 2014. https://doi.org/10.1016/j.polymer.2013.12.062

[8] Jalili, M., Rostami, M., Ramezanzadeh, B. "An investigation of the electrochemical action of the epoxy zinc-rich coatings containing surface modified aluminum nanoparticle", Applied Surface Science, 328, pp. 95-108, 2015.

https://doi.org/10.1016/j.apsusc.2014.12.034

[9] Pi, P., Liu, C., Wen, X., Zheng, L., Xu, S., Cheng, J. "Improved performance of aluminum pigments encapsulated in hybrid inorganic-organic films", Particuology, 19, pp. 93-98, 2015. https://doi.org/10.1016/j.partic.2014.05.010

[10] Iler, R. K., E. I. du Pont de Nemours and Company "Product comprising a skin of dense, hydrated amorphous silica bound upon a core of another solid material and process of making same", USA, US 2885366, 1959.

[11] Karlsson, P., Palmqvist, A. E. C., Holmberg, K. "Surface modification for aluminium pigment inhibition", Advances in Colloid and Interface Science, 128-130, pp. 121-134, 2006. https://doi.org/10.1016/j.cis.2006.11.010

[12] Kiehl, A., Greiwe, K. "Encapsulated aluminium pigments", Progress in Organic Coatings, 37(3-4), pp. 179-183, 1999. https://doi.org/10.1016/S0300-9440(99)00075-2
[13] Kiehl, A., Brendel, H. "Corrosion inhibited metal pigments", Macromolecular Symposia, 187(1), pp. 109-120, 2002.

https://doi.org/10.1002/1521-3900(200209)187:1<109::AIDMASY109>3.0.CO;2-L

[14] Niroumandrad, S., Rostami, M., Ramezanzadeh, B. "Corrosion resistance of flaky aluminum pigment coated with cerium oxides/ hydroxides in chloride and acidic electrolytes", Applied Surface Science, 357, pp. 2121-2130, 2015.

https://doi.org/10.1016/j.apsusc.2015.09.195

[15] Khelifa, F., Druart, M. E., Habibi, Y., Bénard, F., Leclère, P., Olivier, M., Dubois, P. "Sol-gel incorporation of silica nanofillers for tuning the anti-corrosion protection of acrylate-based coatings", Progress in Organic Coatings, 76(5), pp. 900-911, 2013. https://doi.org/10.1016/j.porgcoat.2013.02.005

[16] Edouk, U., Suleiman, R., Khaled, M., Akid, R. "Enhancing water repellency and anticorrosion properties of a hybrid silica coating on mild steel", Progress in Organic Coatings, 93, pp. 97-108, 2016. https://doi.org/10.1016/j.porgcoat.2016.01.006

[17] Oubaha, M., Varma, P. C. R., Duffy, B., Gasem, Z. M., Hinder, S. J. "Development of a Novel Hybrid Aluminium-Based Sol-Gel Materials: Application to the Protection of AA2024-T3 Alloys in Alkaline Environment", Advanced in Materials Physics and Chemistry, 4(5), pp. 75-84, 2014. https://doi.org/10.4236/ampc.2014.45010

[18] Sepeur, S. "Nanotechnology: Technical Basics and Applications", Vincentz Network, Hannover, Germany, 2008.

[19] Álvarez, D., Collazo, A., Nóvoa, X. R., Pérez, C. "Assessment of $\mathrm{ZnO}$ nanoparticles as anticorrosive pigment in hybrid sol-gel film", Progress in Organic Coatings, 96, pp. 3-2, 2016. https://doi.org/10.1016/j.porgcoat.2015.10.017

[20] Ma, Z. L., Li, C. C., Wei, H. M., Ding, D. Q. "Silica sol-gel anchoring on aluminium pigments surface for corrosion resistance based on aluminium oxidized by hydrogen peroxide", Dyes and Pigments, 114, pp. 253-258, 2015. https://doi.org/10.1016/j.dyepig.2014.11.018

[21] Ma, Z. L., Wei, H. M., Li, C. C., Yang, P. F. "Silica sol-gel anchoring on aluminum pigments surface for corrosion protection based on aluminum oxidized by copper ammonia complex ion", Dyes and Pigments, 113, pp. 730-736, 2015. https://doi.org/10.1016/j.dyepig.2014.10.012

[22] Alba Aluminiu "Aluminum Pigments", [online] Available at: http://www.albaaluminiu.ro/ [Accessed: 02 June 2019]

[23] Kim, B., Hochella, M. F. "Chapter 4 - Analytical Transmission Electron Microscopy and Scanning Transmission Electron Microscopy Techniques for the Characterization of Nanomaterial Composition, Phase and Crystallinity", Frontiers of Nanoscience, 8, pp. 124-152, 2015. https://doi.org/10.1016/B978-0-08-099948-7.00004-X

[24] Wang, H., Huang, S., Zuo, Y., Zhou, T., Zhang, L. "Corrosion resistance of lamellar aluminium pigments coated by $\mathrm{SiO}_{2}$ by solgel method", Corrosion Science, 53(1), pp. 161-167, 2011. https://doi.org/10.1016/j.corsci.2010.09.012 
[25] Ma, Z., Qiao, Y., Wen, Y., Wang, X., Wang, J. "Effect of precursors on the anti-corrosion property of silica encapsulated waterborne aluminium pigments", Pigment \& Resin Technology, 46(2) pp. 100-106, 2017. https://doi.org/10.1108/PRT-02-2016-0017

[26] Zhu, H., Qu, X., Hu, Y., Xie, H., Chen, Z. "Corrosion inhibition of flaky aluminum powders prepared through sol-gel process", Corrosion Science, 53(1), pp. 481-486, 2011.

https://doi.org/10.1016/j.corsci.2010.09.062
[27] Li, L., Pi, P., Wen, X., Cheng, J., Yang, Z. "Optimization of sol-gel coatings on the surface of aluminum pigments for corrosion protection", Corrosion Science, 50(3), pp. 795-803, 2008.

https://doi.org/10.1016/j.corsci.2007.11.002 